\title{
Bi-Modal Detection of Painful Reaching for Chronic Pain Rehabilitation Systems
}

\author{
Temitayo A. Olugbade ${ }^{1}$, M. S. Hane Aung ${ }^{1}$, Nadia Bianchi-Berthouze ${ }^{1}$, \\ Nicolai Marquardt ${ }^{1}$, Amanda C. Williams ${ }^{2}$, \\ ${ }^{1}$ University College London, UCL Interaction Centre, Gower Street, London, WC1E 6BT \\ ${ }^{2}$ University College London, Dept. Clinical Psychology, Torrington Place, London, WC1E 6BT \\ \{temitayo.olugbade.13, m.aung, n.marquardt, amanda.williams, n.berthouze\}@ucl.ac.uk
}

\begin{abstract}
Physical activity is essential in chronic pain rehabilitation. However, anxiety due to pain or a perceived exacerbation of pain causes people to guard against beneficial exercise. Interactive rehabiliation technology sensitive to such behaviour could provide feedback to overcome such psychological barriers. To this end, we developed a Support Vector Machine framework with the feature level fusion of body motion and muscle activity descriptors to discriminate three levels of pain (none, low and high). All subjects underwent a forward reaching exercise which is typically feared among people with chronic back pain. The levels of pain were categorized from control subjects (no pain) and thresholded self reported levels from people with chronic pain. Salient features were identified using a backward feature selection process. Using feature sets from each modality separately led to high pain classification F1 scores of 0.63 and 0.69 for movement and muscle activity respectively. However using a combined bimodal feature set this increased to $\mathrm{F} 1=0.8$.
\end{abstract}

\section{Categories and Subject Descriptors}

H.1.2. Human Information Processing

\section{General Terms}

Algorithms, Experimentation, Human Factors.

Keywords: Emotion; Machine Learning; Motion Capture; Electromyography; Body Movement; Pain Rehabilitation Technology; Physical Rehabilitation.

\section{INTRODUCTION}

Interactive technology developed to motivate physical activity has been efficacious in the rehabilitation of chronic conditions [1]. However, for the specific case of chronic pain (CP), efficacy is more limited [2, 3]. One major reason is that rehabilitation technology is not designed to adapt to the emotional state of its user. The person's emotional state is important in CP because pain uniquely conveys threat [4], which generates fear, low confidence or catastrophic thinking leading to avoidance and self-protective behavior [5, 6]. In turn, this greatly reduces adherence to beneficial activity and leads to no progress or even negative effects. In CP rehabilitation, it is well understood that keeping active protects against weakening and stiffness, it inhibits the neurophysiological mechanisms underlying the spread of pain,

Permission to make digital or hard copies of part or all of this work for personal or classroom use is granted without fee provided that copies are not made or distributed for profit or commercial advantage and that copies bear this notice and the full citation on the first page. Copyrights for third-party components of this work must be honored. For all other uses, contact the Owner/Author

Copyright is held by the owner/author(s).

ICMI'14, Nov 12-16 2014, Istanbul, Turkey.

ACM 978-1-4503-2885-2/14/11.

http://dx.doi.org/10.1145/2663204.2663261 increases confidence in physical ability, underpins achieving goals [7] and can improve quality of life [8]. Physiotherapists (and other healthcare staff) regularly educate and advise on activity and provide psychological support to encourage adherence. In Singh et al. [9], the authors discuss how physiotherapists make use of behavioral cues (such as guarding, avoidance, alteration of breathing or anxiety) from the patient to decide on the timeliness, amount and type of support to provide. This can vary from simple breathing prompts to partitioning the exercise to facilitate graded exposure, or simply providing information, reassurance or encouragement. However, limited human resource means there is a heavy reliance on self-management and self-rehabilitation where the psychological states are not observed and thus the aforementioned adherence issues occur [10].

Clearly, automated systems that have the capability to recognize such affective states are a potential solution. However, the development of recognition models to reliably detect pain expression or pain-related behavior remains a complex challenge. To date, the main expressive modality that has been researched is facial expression [11-16]. However, for the design of $\mathrm{CP}$ rehabilitation systems, expression from body movement is essential since many of the protective behaviors are manifest in whole-body motion [6]. To the best of our knowledge, automatic detection of pain-related expressions from body movement have barely been studied [17]; although in the wider context of generic affective states, there has been much recent work on automatic recognition from body movements and postures (for a review see [18]) and in sports-games [19]. In addition to outward expressions, another measure that reflects protective behavior is anomalous levels of muscle activity given a particular movement context. In a surface electromyographic (sEMG) study specific to chronic low back pain (CLBP), Watson et al. [20] showed that psychological factors influenced the prolonged use of lumbar paraspinal muscles during forward flexion exercises. Similarly, in a study by Van der Hulst et al. [21], the authors showed that there was an association between high sEMG levels and a guarded mechanism during walking. In Geisser et al. [22], the authors examined the relationship between lumbar flexion, dynamic sEMG and pain-related fear in people with CLBP. Correlations were found between pain-related fear and reduced lumbar flexion as well as increased sEMG during full flexion.

In this study, we develop and evaluate a recognition framework to automatically classify forward reaching motions (Fig. 1) in people without chronic pain, and people with chronic pain reporting low pain or high pain after the execution of the exercise. We used supervised machine learning with early fusion of features processed from whole body motion capture (MoCap) and sEMG data from the lumbar paraspinal and upper trapezius muscles. In Section 2, we describe the nature of the dataset we used and its 
acquisition method. In Section 3, we describe the initial feature set identified as potentially descriptive of pain based on literature and initial inspection. In Section 4, we report the results of an ANOVA analysis of the features and we also report on the optimization and validation of three Support Vector Machine (SVM) frameworks using three initial feature sets: two frameworks were trained using unimodal features (MoCap and sEMG respectively) and the third using a combined bimodal feature set. We conclude in Section 5 by discussing how these findings inform current development of automatic CP rehabilitation technology.

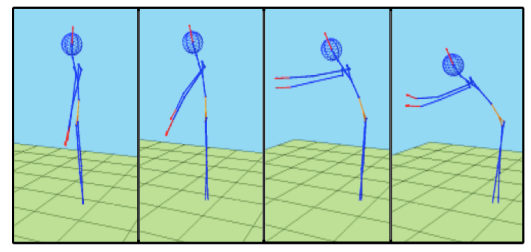

Figure 1. Example of a forward phase of the reach exercise

\section{DATASET DESCRIPTION}

The data used in this study is a subset of the extensive multimodal database presented in [23], which contains a wider set of movements and sensing modalities and pain-related labeled behaviors. The subset considered here consists of 18 people with CLBP and 13 control subjects undergoing forward-reaching motions (Fig. 1), this yields 30 instances of reaching from the CLBP group and 19 instances from the control group. This exercise is part of rehabilitation routines in CLBP and is one that generates anxiety and fear possibly due to a perceived exacerbation of pain [9].

To capture movement the subjects wore a suit with Inertial Measuring Units (IMUs) (Animzaoo IGS-190). Each IMU was attached with a minimal amount of velcro-strapping to reduce invasiveness. Each IMU returned 3D Euler angle triplets in the BioVision Hierarchy format $(\mathrm{BVH})$ at a sample rate of $60 \mathrm{~Hz}$. In conjunction, skeletal proportions were annotated from still photographs of each subject. Finally, 3D Cartesian positional triplets of 26 anatomical nodes over the whole body were calculated. Also, 4 wireless electromyographic (sEMG) sensors (BTS FREEEMG) were attached to the lumbar paraspinal muscles (hereafter, called lower-back muscles) and to the upper section of the trapezius muscles (hereafter, called upper-back muscles). Each pair of sensors was placed laterally equidistant to the spine and sampled at $1 \mathrm{kHz}$. The recording procedure of the two sensing systems was automatically triggered for synchronization.

Each instance of a reached forward movement consisted of a forward reach with both hands while standing, then a retraction back to a standing pose (Fig. 1). After each instance, the CLBP participants were asked to report a level of pain defined as a value between 0 for no pain to 10 for extreme pain (mean 3.85, standard deviation 3.16). Within the CLBP group, instances were categorized as low pain if the self reported rating was $<5$ and high pain if $\geq 5$. This resulted in 15 instances in the low pain group and 15 in the high pain group. The 19 control instances are categorized as no pain. Overall, this is treated as a 3 category classification task for two main reasons. First, the control instances are not equivalent to CLBP instances with zero reported pain, control subjects will have no fear of pain exacerbation and no fear of performing the exercise and [5] are therefore a separate class. Secondly, self reporting is highly subjective and variable between subjects and a broad categorization is less dependent on the reported values compared to a regression task.

\section{FEATURE EXTRACTION}

In this section, we describe the feature set derived from MoCap and sEMG information. Due to the large amount of information that can be potentially processed from whole body MoCap and sEMG signals, we aimed to find a minimal feature set salient to the reaching forward movement.

Movement Features. With the rationale that pain intensity affects performance in physical exercise [24], we visually inspected the movement profiles of various body segments during each reaching instance. It was found that the vertical displacement of the forearm and the neck and flexion angles showed differences between the two pain level groups. We consider five features derived from these profiles as described in Table 1 (features 1 5). To account for differences in skeletal proportions, positional features were normalized accordingly.

Muscle Activity Characteristics. In concordance with [20] and [22], sEMG signal characteristics during a forward flexion movement shows typically high activity at the onset to apex phase followed by a significant decrease in activity during retraction. Fig. 2-left shows this expected sEMG profile with a drop in activation during retraction after the change point (indicated by a red spot). Fig 2-right shows an example of a lack of change in muscle activation due to poor execution or avoidance in performing the movement. Using the upper envelope of the rectified signal, we automatically extracted the change point, i.e. the approximate time at which the highly active onset to apex phase changes to the retraction phase with low activity. This was done by adapting the method in [25] using two equal-sized sliding windows (50-frame in width) with a 20 -frame separation sliding across the whole signal. The twin-window location that optimizes the difference between the mean activities within the two windows was found. The middle frame value between the two windows normalized by the duration of the whole motion determines the feature ChangePointTimeRatio. Also the signed maximum difference in mean activity from the two windows normalized by the maximum activity of the whole motion yields the feature ChangePointDifference. These quantities were calculated for all four sEMG channels (see features $6-13$ in Table 1). Pain-related waveforms with relatively high activity in the retraction phase gave lower differences or even negative values and occurred at irregular times.
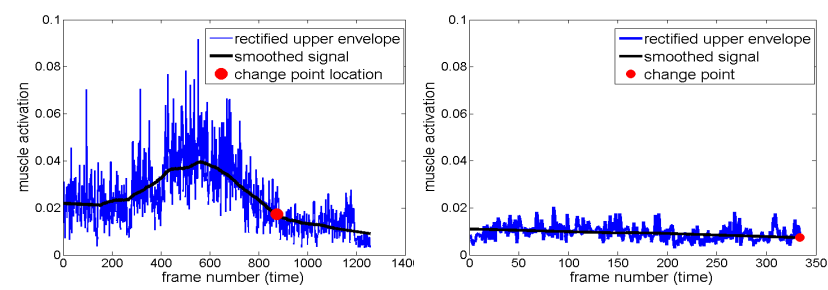

Figure 3. Lower back sEMG signals from a reaching motion with change point locations, normal pattern (left) with avoidance (right).

\section{MODELLING AND RESULTS}

An initial one-way analysis of variance (ANOVA) was carried out, using SPSS v.21, to test the statistical significance of each of the feature with the three levels of pain. We use the left arm only due to lateral symmetry thus avoiding the duplication of values and reducing feature dimensionality. The results showed that most of the features were statistically significant (see bold p- values in Table 1). 
Table 1: Features and anatomical nodes (highlighted in yellow on the stick figure) used in the study. For clarity, TopNeck $=24$, LowNeck = 12, Hip = 1, UpperRightLeg = 7, UpperLeftLeg $=2$, LowerRightLeg $=8$, LowerLeftLeg $=3$, RightFoot $=9$, LeftFoot $=$ 4, UpperLeftArm=15, LowerLeftArm = 16.

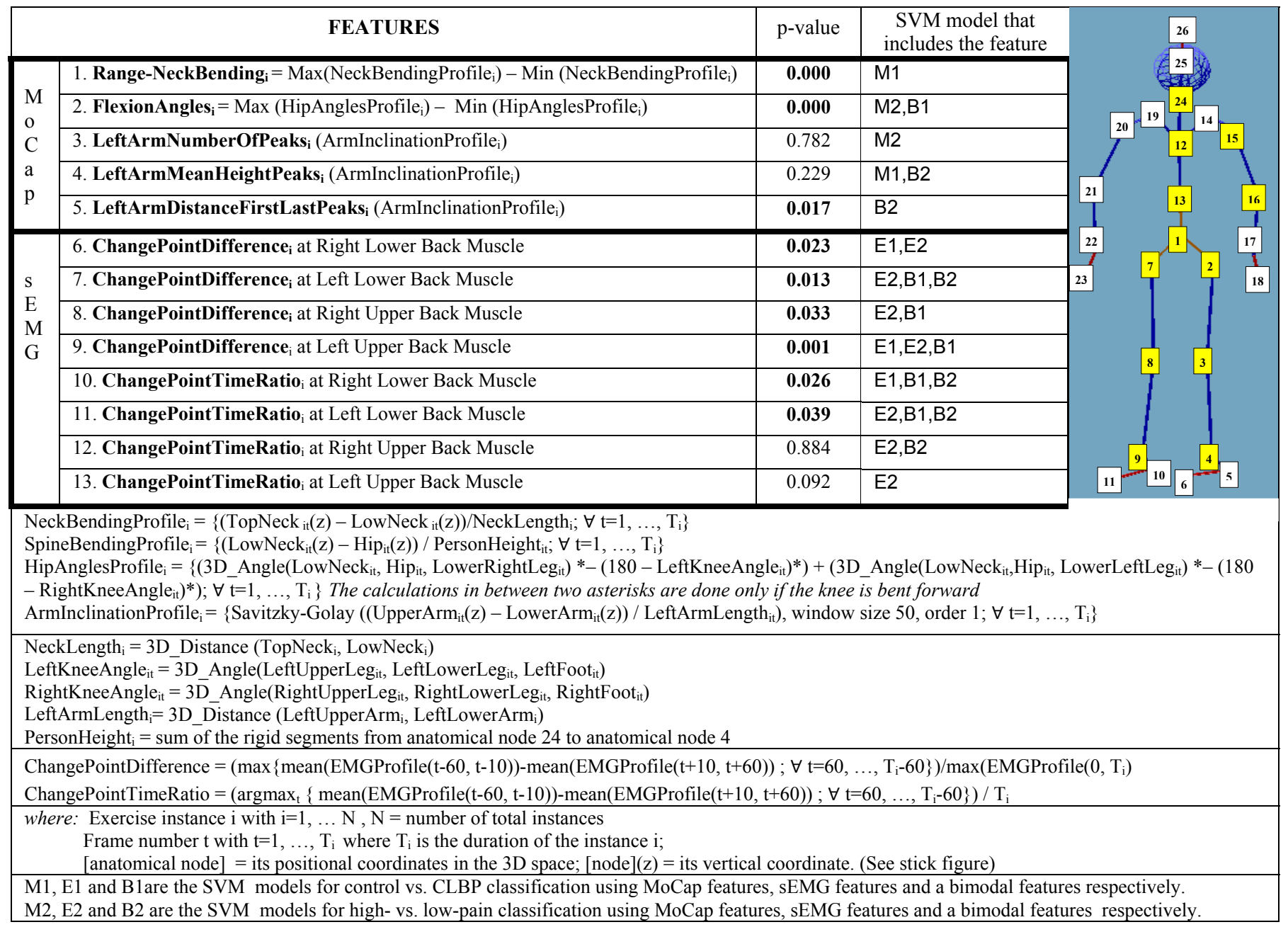

Table 2. Confusion Matrices showing number of instances.

\begin{tabular}{|c|c|c|c|c|}
\hline & \multicolumn{4}{|c|}{ M1+ M2 models: Predicted using MoCap features only } \\
\hline & & Control & Low-Pain & High-Pain \\
\hline \multirow{5}{*}{ 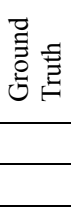 } & Control & $13(68.4 \%)$ & $1(5.3 \%)$ & $5(26.3 \%)$ \\
\hline & Low-Pain & $0(0 \%)$ & $11(73.3 \%)$ & $4(26.7 \%)$ \\
\hline & High-Pain & $0(0 \%)$ & $4(26.7 \%)$ & $11(73.3 \%)$ \\
\hline & \multicolumn{4}{|c|}{ E1+ E2 models: Predicted using sEMG features only } \\
\hline & & Control & Low-Pain & High-Pain \\
\hline \multirow{5}{*}{ 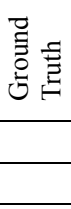 } & Control & $19(100 \%)$ & $0(0 \%)$ & $0(0 \%)$ \\
\hline & Low-Pain & $0(0 \%)$ & $13(86.7 \%)$ & $2(13.3 \%)$ \\
\hline & High-Pain & $2(13.3 \%)$ & $4(26.7 \%)$ & $9(60 \%)$ \\
\hline & \multicolumn{4}{|c|}{ B1 + B2 models: Predicted using Bi-modal features } \\
\hline & & Control & Low-Pain & High-Pain \\
\hline \multirow{3}{*}{ 总吉 } & Control & $17(89.4 \%)$ & $1(5.3 \%)$ & $1(5.3 \%)$ \\
\hline & Low-Pain & $0(0 \%)$ & $13(86.7 \%)$ & $2(13.3 \%)$ \\
\hline & High-Pain & $0(0 \%)$ & $3(20 \%)$ & $12(80 \%)$ \\
\hline
\end{tabular}

Next, we investigated the use of a hierarchically-structured SVM framework with two levels. This was done to create an overall three class model: no pain (control instances), low pain and high pain. The SVMs M1, E1 and B1 in level 1 of the hierarchy are trained to discriminate control instances from CLBP instances. If an instance is classed as belonging to the CLBP group, this invokes an SVM in level 2 referred to as M2, E2 or B2 trained to discriminate between high-pain and low-pain instances. We test 3 initial feature sets: MoCap only (M-models: features 1-5), sEMG only (E-models: 6-13) and bi-modal (B-models: 1-13).

For each initial set, we apply a backward feature selection process to prune indiscriminative features and reduce dimensionality. Subject dependent cross-validation (leave-one-subject-out) was applied to the SVM at each level of the hierarchy, which included an evaluation subject to test three different kernels (linear, polynomial and hyperbolic-tangent) as well as grid searching over a range of kernel parameters and the SVM regularisation parameter $C$. We obtain the following optimal models: MoCap only level 1 (M1) - hyperbolic-tangent kernel with coefficients 0.01 and $-0.01, C=0.01$, MoCap only level 2 (M2) hyperbolictangent kernel with coefficients 10 and $-10, C=10$; sEMG only level 1 (E1) - linear with $C=1$, sEMG only level 1 (E2) hyperbolic-tangent kernel with coefficients 1 and $-1, C=10$; bimodal level 1 (B1) quadratic kernel with $C=1$, bi-modal level 2 (B2) -hyperbolic-tangent kernel with coefficients 1 and -1 and $C$ $=1$. The third column in Table 1 shows which model utilized the 
described feature as a result of the backward selection. For the MoCap-only experiment, we obtain an overall F1 score of 0.63 with the high pain class as the positive class and an accuracy of 0.71. The sEMG-feature-based model gave a F1 score of 0.69 with an accuracy of 0.84 . Using the bi-modal feature set, an F1 score of 0.80 and an accuracy of 0.86 were obtained. Table 2 shows the confusion matrices of the classified instances.

\section{DISCUSSION}

This study investigated the use of body movement and muscle activity features to detect perceived pain level in a typical exercise used in CLBP physical rehabilitation: reaching forward. The findings show good performance in separating healthy people from CLBP people using bi-modal data or with sEMG only. But to discriminate between low and high perceived pain levels, the use of bi-modal data yields the optimal performance. However, further optimization could include fusion at a decision level which could model the underlying distributions per modality in a better way. The ANOVA tests on each feature and the backward selections show lower back activity and forward flexion angle at the hip to be very important and to a lesser extent bending at the neck and upper back muscle activity. An understanding of important anatomical locations can inform the design of future wearable rehabilitation technology. Such outcomes are timely since unobtrusive low-cost MoCap systems are becoming more accurate and robust. Additionally, new smaller and wearable sEMG sensors make muscle activity measurement more affordable for home-based rehabilitation. In addition, it would be useful to extend this work to other typical exercises to understand the generalizability of the features. For CP rehabilitation, the robust recognition of painful motion can be used in personalized rehabilitation technology [26]. Being able to detect perceived pain would allow the tailoring of the rehabilitation technology to the psychological and physical skills of the patient as described in [9].

\section{REFERENCES}

[1] Lin, J., Mamykina, L., Lindtner, S., Delajoux G. and Strub H. 2006. Fish'n'Steps: Encouraging Physical Activity with an Interactive Computer Game, UbiComp, LNCS 4206 261278.

[2] Schonauer, C., Pintaric, T., Kaufmann, H., Jansen, S. and Vollenbroek-Hutten M. 2011. Chronic pain rehabilitation with a serious game using multimodal input. Proc. ICVR, 1-8

[3] Cepeda, M., Carr, D., Lau, J., Alvarez, H. 2006. Music for pain relief. Cochrane Database of Syst. Rev. 2.

[4] Crombez, G., Eccleston, C., Van Damme, S., Vlaeyen, J., and Karoly, P. 2012. The fear avoidance model of chronic pain: the next generation. Clin J Pain 28, 475-483.

[5] Vlaeyen, J. and Linton, S. 2000. Fear-avoidance and its consequences in chronic musculoskeletal pain: a state of the art. Pain 85(3).

[6] Keefe, F. and Block, A. 1982. Development of an observation method for assessing pain behaviour in chronic low back pain patients. Behaviour Therapy 13(4), 363-375.

[7] Gatchel, R., Peng, Y., Peters, M. and Turk D. 2007. The biopsychosocial approach to chronic pain: scientific advances and future directions. Psychol Bull 133(4), 581-624

[8] Williams A. C. de. C., Eccleston, C, and Morley, S. 2012. Psychological therapies for the management of chronic pain (excluding headache) in adults. Cochrane Database of Systematic Reviews, Issue 11. Art. No.CD007407
[9] Singh, A., Klapper, A., Swann-Sternberg, T, et al. 2014. Motivating People with Chronic Pain to do Physical Activity: Opportunities for Technology Design, ACM ,CHI' 2014.

[10] Turk, D. and Okifuji, A. 2002. Psychological factors in chronic pain: evolution and revolution. J Consult Clin. Psychol. 70(3), 678-690.

[11] Romera-Paredes, B., Aung, M., Pontil, M., Watson, P., Williams, A CdeC, and Bianchi Berthouze, N. 2013. Transfer Learning to Account for Idiosyncrasy in Face and Body Expressions. $10^{\text {th }}$ IEEE Int. Conf. Auto. Face \& Gesture Rec.

[12] Littlewort, G., Barlett, M., and Lee, K. 2009. Automatic coding of facial expressions displayed during posed and genuine pain. Image Vision Comp 27(12), 1797-1803.

[13] Niese, R., Al-Hamadi' A., Panning, A. et al. 2009. Towards Pain Recognition in Post Operative Phases using 3D based features from Video and Support Vector Machines. Int $J$. Digital Content Tech. App. 3(4).

[14] Rudovic, O., Pavlovic, V., and Pantic, M. 2013. Automatic Pain Intensity Estimation with Heteroscedastic Conditional Random Fields. Adv. in Vis. Comp., Springer. 8034, 234-243.

[15] Kaltwang, S., Rudovic, O., and Pantic, M. 2012. Continuous Pain Intensity Estimation from Facial Expressions. Adv. in Vis. Comp., Springer, Heidelberg, 7432, 368-377.

[16] Hammal, Z., and Cohn, J. F. 2012. Automatic detection of pain intensity. $14^{\text {th }}$ Int. Conf. Multimodal Interaction, 47-52.

[17] Aung, M., Romera-Paredes, B., et al. 2013. Getting rid of pain related behaviour to improve social and self perception: a technology based perspective. $14^{\text {th }}$ Int. Workshop on Image. Anal. Multimedia Interactive Services.1-4.

[18] Kleinsmith, A., and Bianchi-Berthouze, N. 2013. Affective Body Expression Perception and Recognition: A Survey. IEEE Trans. on Affective Computing 4(1), 15-33.

[19] Savva, N., Scarinzi, A., and Bianchi-Berthouze, N. 2012. Continuous Recognition of Player's Affective Body Expression as Dynamic Quality of Aesthetic Experience. IEEE Trans. CIAIG 3, 199-212.

[20] Watson, P., Booker, K., and Main, C. 1997. Evidence for the role of Psychological Factors in Abnormal Paraspinal Activity in Patients with Chronic Low Back Pain. $J$. Musculoskelet. Pain 5(4), 41-56.

[21] Van der Hulst, M., Vollenbroek-Hutton, M., Reitman, J. et al. 2010. Back Muscle Activation Patterns in Chronic Low Back Pain during Walking: A "Guarding” Hypothesis. Clin J Pain 26(1), 30-37

[22] Geisser, M., Haig, A., Wallbom, A., and Wiggert, E. 2004. Pain-related fear, lumbar flexion, and dynamic SEMG among persons with chronic musculoskeletal low back pain. Clin $J$ Pain. 20(2), 61-69

[23] Aung, M.S.H., Kaltwang S., et al. 2014. Detecting Chronic Pain-Related Expressions and Behaviours from Multimodal Naturalistic Data. IEEE Trans. on Affective Computing (in submission).

[24] Rainville, J., Ahern, D., Phalen, L. et al. 1992. The Association of Pain with Physical Activities in Chronic Low Back Pain. Spine 17(9), 1060-1064.

[25] Marple-Horvat, D. E. and Gilbey, S. L. 1992. A method for automatic identification of periods of muscular activity from EMG recordings. J Neurosci Mtds 42(3), 163-167.

[26] Jensen, M., Turner, J., and Romano, J. 2001. Changes in beliefs, catastrophizing and coping are associated with improvement in multidisciplinary pain treatment. J Cons Clin Psychol 69(4), 655-662. 